that there are tests of stellar atmospheres modeling that result from observations of nebulae associated with hot stars, and which are usually overlooked. We illustrate this point with a specific example where there is a persistent problem matching certain observed nebular emission line fluxes with photoionization models of $\mathrm{H}$ il regions. Evidence is presented for what is called the [ $\mathrm{Ne} \mathrm{III]} \mathrm{problem} \mathrm{-} \mathrm{that} \mathrm{is,} \mathrm{the} \mathrm{H}$ II region models predict significantly less flux in the [Ne III] lines than is measured. The predicted nebular fluxes are affected not only by the representation of the stellar emergent ionizing spectra but also by the prodigious set of atomic data, that is an input ingredient in such calculations. However, it is argued that difficulties with matching [Ne III] fluxes are most likely due to the use of LTE stellar atmosphere models to represent the hot stars ionizing the observed $\mathrm{H}$ II regions.

Post-meeting calculations utilizing atmospheres by Kunze that do not assume LTE permit us to directly test the above conjecture. When these new atmospheres are used in the model nebula code, there are significantly enhanced [Ne III] fluxes compared with those run using the LTE atmospheres. When we consider a reasonable range of nebular parameters as well as reasonable expectations for Kunze models with effective temperature $T_{\text {eff }}=35000$ $40000 \mathrm{~K}$, the [Ne III] problem is likely resolved. As an additional benefit of the model nebula calculations with Kunze non-LTE and Kurucz LTE atmospheres, we are able to also examine several criteria that have been used to estimate a characteristic $\mathrm{T}_{\text {eff }}$ for $\mathrm{H}$ II regions. Three indicators of "ionization" based on measured line ratios are examined. Two are from far-infrared data: [O III] $52 \mu \mathrm{m} /[\mathrm{S}$ III] $33 \mu \mathrm{m}$ and $[\mathrm{N} \mathrm{III]} 57 \mu \mathrm{m} /[\mathrm{N} \mathrm{II}] 122 \mu \mathrm{m}$; the other is the ratio of adjacent radio recombination lines of helium and hydrogen. For a given line ratio in any of these three cases, the $T_{\text {eff }}$ inferred will be lower for the non-LTE stellar atmosphere compared with the LTE one. Likewise, a non-LTE stellar atmosphere will be more highly ionized than the corresponding (same $\mathrm{T}_{\text {eff }}$, surface gravity, and composition) LTE atmosphere.

\title{
OPACITIES IN STRONG MAGNETIC FIELDS
}

\section{DAYAL T. WICKRAMASINGHE}

The Astrophysical Theory Centre, Australian National University, Canberra ACT 2601, Australia

White dwarfs are one of the most readily studied end products of stellar evolution. Their observed properties have provided and continue to provide important constraints for the theory of stellar evolution. Likewise, a study of magnetism in white dwarfs provides unique insights into the origin and evolution of magnetic fields in stars.

Spectacular progress has been made on the specific problem of the structure of the 
hydrogen atom in strong fields. Energy levels and transition probabilities are now known for all low lying states of hydrogen for the entire range of field strengths appropriate to white dwarfs and neutron stars $\left(10^{4}-10^{13} \mathrm{G}\right)$ (Rosner et al 1984, Forster et al 1984 and Henry and O'Connell 1984). These calculations resulted in the identification of spectral features in the magnetic white dwarf $\mathrm{Grw}+70^{\circ} 8247$ which had remained unidentified for over 50 years (Minkowski 1938), with Zeeman shifted hydrogen lines in a magnetic field of $100-320$ MG ((eg Wickamasinghe and Ferrario 1989). Several other strong field magnetic white dwarfs have since been discovered through hydrogen Zeeman spectroscopy. The data presently at hand show that most hydrogen rich magnetic white dwarfs have complex non-dipolar field structures with strong evidence for higher order multipole components.

It is well established that the atmospheres of non magnetic white dwarfs are monoelemental, consisting either of essentially pure hydrogen or pure helium. It is likely that a similar situation will pertain to the magnetic white dwarfs. The next major step ahead, therefore, would be the identification of spectral features in strongly polarised white dwarfs with helium transitions in strong magnetic fields. There are several stars estimated to have strong fields from their polarisation properties and which exhibit spectral features which have so far defied explanation. The best known example is GD229 which shows broad and deep features almost equally spaced in wavelength extending from the optical to the far UV. Accurate calculations of the bound energy levels and transition probabilities for $\mathrm{HeI}$ in the field range $10^{8}-10^{9}$ Gauss, where Coulomb and magnetic terms play comparable roles in the Hamiltonian are still unavailable, although considerable progress had been made on this problem recently (Thurner et al 1993). It is clear that enigmatic stars such as GD229 will eventually provide new insights on the physics of atoms in strong magnetic fields, in regimes that are not readily accessible to terrestial laboratories.

The input physics required to calculate realistic model stellar atomspheres for strongly magnetic white dwarfs is at the best only poorly understood. One of the major obstacles has been the intrinsic difficulty in carrying out atomic physics calculations of energy levels and transition probabilities in strong magnetic fields in the regime of mixed (spherical and cylindrical) symmetries. Where both Coulomb and magnetic terms are of comparable importance. The effect that a magnetic field has on even the commonest sources of continuum opacity, such as bound - free and free - free absorption due to $\mathrm{H}, \mathrm{HeI}, \mathrm{HeII}$ and $\mathrm{H}^{-}$is at present not well understood.

An important advance has recently been made in the calculation of bound - free opacities due to hydrogen in strong magnetic fields (Merani et al 1994). These calculations show that the Landau continua have embedded in them resonances which give the opacity a complex wavelength structure which may be detected as spectral features in the observations. The bound-free results need to be extended to a wider field range before they can be useful for the interpretation of white dwarfs in the optical spectra.

A similar advance is urgently required for free - free opacity in a strong magnetic field (magneto-bremsstrahlung). A full quantum treatment of this opacity is presently unavailable 
except at the cyclotron resonance. However, additional resonances resulting from the Coulomb interaction may also contribute to this opacity and these may, under certain circumstances, appear as continuum features in the optical spectra.

Perhaps the greatest challenge, however, would be the construction of realistic model atmospheres and line profiles for magnetic white dwarfs at arbitrary field strengths at a level of sophistication that will enable effective temperatures and gravities to be determined from observations. This requires, in addition, the development of a theory of Stark broadening in strong magnetic fields.

Forster, H., Strupat, W., Rosner, W., Wunner, G., Ruder, H. and Herold, H., 1984, J.Phys. B; At. Mol Phys. 17, 1301

Henry, R.J. W. and O'Connell, R.F., 1984, ApJ, 282, L28

Minkowski, R., 1938, Ann. Rept. Div. Mt. Wilson Obs., 28

Merani, N., Main, J. and Wunner, G., A\&A, in press

Rosner, W., Wunner, G., Herold, H. and Ruder, H., 1984, J. Phys. B.; At. Mol. Phys., 17, 29

Thurner, G., Korbel, H. Braun, M., Herold, H., Ruder, H. and Wunner, G., 1993, J. Phys. B.; At. Mol. Opt. Phys., 26, 4719

Wickramasinghe, D.T. and Ferrario, L., 1988, ApJ, 327, 222

Third Session: Stellar Structure (Tuesday August 23, afternoon)

HORIZONTAL BRANCH EVOLUTION AND RR LYRAE STAR PULSATION

\author{
ARTHUR N. COX \\ Los Alamos Astrophysics, Los Alamos National Laboratory, MS B288 \\ P.O. Box 1663, Los Alamos, NM 87545 USA
}

For many years there has been a confrontation between stellar evolution and pulsation theories concerning the masses, luminosities, and compositions of the horizontal branch RR Lyrae variables. Masses obtained by Cox, Hodson \& Clancy (CHC, 1983) were very low, but Kovacs (1985) and later Kovacs \& Buchler (1988) suggested somewhat larger ones. Even later Simon \& Cox (1991) verified CHC results, though still using the Los Alamos opacities. Petersen $(1991,1992)$ has also discussed this mass problem in some detail. The persistent discrepancy of $0.1 \mathrm{M} \odot$ or more between the evolution and pulsation masses was mostly ignored because neither theory could find any significant flaw in its analysis. Cox (1991), Kovacs, Buchler \& Marom (1991), and Kovacs, Buchler, Marom, Iglesias \& Rogers (1992) finally showed that larger double-mode pulsation masses, are consistent with evolution 